ArtefaCToS. Revista de estudios de la ciencia y la tecnología

eISSN: 1989-3612

Vol. 7, No. 1 (2018), 2a Época, 13-28

DOI: http://dx.doi.org/10.14201/art2018711328

\title{
La transformación neoliberal de la ciencia: El caso de las Humanidades Digitales
}

\author{
The Neoliberal Transformation of Science: The Case of Digital \\ Humanities
}

\author{
Eduard AIBAR PUENTES \\ Universitat Oberta de Catalunya, España \\ eaibar@uoc.edu
}

Recibido: 12/12/2017. Revisado: 20/12/2017. Aceptado: 27/12/2017

\begin{abstract}
Resumen
Los impactos de las políticas y prácticas neoliberales están produciendo la transformación más importante de la ciencia y la academia contemporáneas desde mediados del s. XX. Las políticas científicas neoliberales han puesto el énfasis más en la creación de valor comercial que en la consecución del bienestar social o en la generación de conocimiento; se ha fomentado el uso de patentes más que la difusión abierta del conocimiento y se ha promovido la inversión privada en las universidades y en los proyectos de investigación desarrollados por sus investigadores, con objeto de favorecer aquellas líneas de investigación de mayor aplicación comercial y, por tanto, con mayores expectativas de retorno económico. Este trabajo ofrece, por un lado, una panorámica sintética y estructurada de estas transformaciones, haciendo especial hincapié en aquellos cambios que afectan a los métodos, objetos y productos de la actividad científica. La literatura existente sobre estos temas centra su atención, preferentemente, en las ciencias naturales o "duras" como el ámbito biomédico, así que el segundo objetivo de este trabajo es discutir la emergencia de este tipo de fenómenos en un ámbito mucho menos estudiado: el de las humanidades. En particular nos centraremos en el terreno de las denominadas 'humanidades digitales'.
\end{abstract}

Palabras clave: neoliberalismo; mercantilización del conocimiento; privatización de la ciencia; tecnocentrismo; neutralidad de los datos. 


\begin{abstract}
The impacts of neoliberal policies and practices are producing the most important transformation of contemporary science and academia since the mid twentieth century. Neoliberal scientific policies have placed more emphasis on the creation of commercial value than on the achievement of social welfare or the generation of knowledge; the use of patents has been encouraged more than the open dissemination of knowledge and private investment has been promoted in universities and research projects developed by its researchers, in order to favour those lines of research of greater commercial application and with higher expectations of economic return. This work offers, on the one hand, a synthetic and structured overview of these transformations, with special emphasis on those changes that affect the methods, objects and products of scientific activity. The existing literature on these topics focuses, preferably, on the natural or "hard" sciences — particularly on biomedicine - so the second objective of this work is to discuss the emergence of this type of phenomena in a much less studied area: the humanities. In particular, we will focus on the so-called 'digital humanities'.
\end{abstract}

Keywords: Neoliberalism; Commodification of Knowledge; Privatization of Science; Tecnocentrism; Data Neutrality.

\title{
1. Introducción
}

El neoliberalismo constituye tanto una ideología como un conjunto de prácticas, fundamentalmente económicas y políticas, que está marcando el devenir de las sociedades contemporáneas desde la década de los 80 del siglo pasado y definiendo la nueva versión del capitalismo en la era de la globalización. A diferencia de la visión liberal, en que el mercado se entiende por oposición al Estado, en el paradigma neoliberal el objetivo es introducir la lógica del mercado - la competencia - en todos los ámbitos sociales; no sólo en el económico o productivo, sino en la educación, la cultura, los servicios públicos o la propia experiencia vital. Una tarea para la cual el Estado y las administraciones públicas devienen instrumentos esenciales (Dardot y Laval, 2014; Foucault, 2009).

El liberalismo promueve una retirada (mayor o menor, según las versiones más o menos socialdemócratas de que se trate) del Estado frente al mercado mediante la privatización de los servicios y bienes públicos, fundamentalmente. Para el neoliberalismo, en cambio, aunque también se apoyan e incentivan los procesos de privatización, lo esencial es la extensión de la lógica del mercado a toda la vida social, incluyendo al propio Estado. No se trata, por lo tanto, del simple laissez faire o de limitar la intervención del Estado en la economía, sino de facilitar la intervención política para introducir la lógica de la competencia en to- 
dos los ámbitos posibles y reconfigurar, así, las relaciones sociales y económicas. El Estado deja de ser ese mecanismo casi residual que únicamente debe paliar los efectos sociales nocivos del mercado, para devenir un agente poderoso que debe anular los mecanismos anticompetitivos de la sociedad. El mercado, además, no es ya una institución espontánea o natural: es un ente artificial que debe ser creado y modelado políticamente de forma incesante. No es un mero lugar de intercambio de mercancías, sino un procesador de información: es, por encima de todo, un mercado de ideas y, por tanto, también ¡un fenómeno epistémico!

La unidad morfológica básica de la sociedad neoliberal es la empresa. Se trata, por lo tanto, de construir una trama social en que las unidades fundamentales tengan la forma de la empresa o se comporten y funcionen como si lo fueran. La familia, la universidad, la escuela, el teatro, etc., deben gestionarse como empresas y sus agentes — madres profesoras, investigadoras, creadoras, etc.- han de devenir empresarias o emprendedoras. El componente subjetivo del neoliberalismo se muestra en la figura del emprendedor - y no la del mercader o la del consumidor- que se ve a sí mismo como empresa-marca, como empresario de sí mismo y que adopta como valores esenciales la flexibilidad y la capacidad de adaptación (frente a los cambios del mercado), la destreza para venderse y la habilidad para competir. Como dijo una vez Margaret Thatcher "economics are the method: the object is to change the soul'.

\section{Ciencia y neoliberalismo}

La pregunta que se han planteado diferentes autores (principalmente desde los estudios de ciencia y tecnología, STS) es si el neoliberalismo está afectando de alguna manera a la ciencia contemporánea. La tesis básica de investigadores como Philip Mirowski (2011) es que no sólo la está afectando, sino que, de hecho, hemos entrado desde principios de los 80 , en una nueva fase histórica en la organización de la ciencia y de sus relaciones, con la industria, las finanzas y el Estado.

Las transformaciones a que nos referimos han sido conceptualizadas durante los últimos años con expresiones como capitalismo académico, academia acelerada, capitalismo cognitivo, universidad-empresa o ciencia neoliberal. Todas ellas ponen el énfasis, por un lado, en la creciente subordinación de la ciencia a los intereses privados (principalmente, de empresas y grandes corporaciones) y, por otro, a la reestructuración de la actividad científica en base a la lógica de la competencia y a la comercialización de sus resultados (Pellizzoni y Ylönen, 2012; Pestre, 2003; Radder, 2010; Lave, Mirowski \& Randalls, 2010).

Es necesario recordar, en este sentido, que más de dos tercios de la investigación científica actuales son de carácter privado (OECD, 2015): o bien se llevan a cabo en el seno de empresas y organismos privados, o se desarrollan, con fondos 
privados, dentro de instituciones públicas como las universidades (a través de investigación contratada, doctorados industriales, convenios de colaboración con empresas, etc.). De hecho, se observa un decrecimiento continuo en la financiación pública de la investigación durante las últimas tres décadas. El equilibrio entre ciencia pública y privada, que existió a lo largo de la mayor parte del siglo $\mathrm{XX}$, se ha roto en beneficio de la segunda (David, 2004) y hay que tener en cuenta que la ciencia privada es, en gran parte, ciencia propietaria y cerrada, es decir, que no permite el acceso libre a sus contenidos o resultados, ni su posterior reutilización.

La transformación neoliberal de la ciencia contemporánea afecta especialmente a las universidades, que se conciben y gestionan, cada vez más, como empresas o corporaciones multinacionales (Halffman y Radder, 2015). Los organismos de decisión y de poder en la universidad están pasando del sector académico al administrativo - en EE.UU. desde el 2006, por ejemplo, el porcentaje de personal fijo en el sector administrativo supera al académico en la mayoría de las universidades y, en éste, se aprecia una creciente polarización entre una pequeña élite de profesores bien pagados y con recursos y una mayoría de personal académico precarizado y sin apenas recursos. Se está produciendo, igualmente, una separación creciente entre la función universitaria docente y la investigadora. Las universidades compiten entre sí por captar estudiantes (tratados como "clientes"), las titulaciones se consideran 'productos' a comercializar y el lenguaje y las técnicas propias del management inundan todos los rincones de la academia.

\section{La privatización de la ciencia}

La privatización del conocimiento científico se ha facilitado y acelerado, principalmente, a través del fortalecimiento de las leyes de propiedad intelectual. Se están privatizando, no sólo los resultados, sino las herramientas e instrumentos de investigación (tests, procedimientos de medida, etc.): durante el período 1990-2004 la mitad de las patentes en biotecnología en EE.UU. eran ya de este segundo tipo (Mirowski, 2011).

En general se observa una progresiva subordinación de la investigación a intereses empresariales privados — una situación especialmente notable en el ámbito biomédico. Por poner sólo dos ejemplos, la prestigiosa revista New England Journal of Medicine retiró en 2002 su estricta política sobre el conflicto de intereses por la dificultad creciente de encontrar revisores, para artículos sobre nuevos medicamentos, sin vínculos con la industria farmacéutica. Los denominados autores fantasma - científicos que, a cambio de una suma de dinero, ceden su firma sin haber participado en la investigación- aparecen ya en más del $40 \%$ de este tipo de artículos que devienen, por tanto, verdaderos publirreportajes más que verdaderos estudios científicos (Sismondo, 2009). 
El discurso y la lógica del emprendimiento han colonizado el seno de la investigación académica (Quintanilla, 2012). Las instituciones académicas y los gobiernos promueven cada vez más, la creación de start-ups, spin-offs y los contratos con empresas en el entorno científico. El investigador deviene emprendedor/empresario/gestor - las universidades organizan continuamente cursos de formación para ello- y dedica cada vez más tiempo a promocionar su "propia marca" a través de las redes sociales privadas (Twitter, Facebook, etc.) o de las nuevas redes sociales "académicas" (Research.Gate, Academia.edu, Mendeley, etc.), también privadas. El mundo de la publicación académica está también fuertemente privatizado. Unas pocas empresas multinacionales (Elsevier, Springer, Wiley-Blackwe11, Taylor \& Francis y Sage) controlan más de la mitad de revistas científicas en todo el mundo - hasta hace pocas décadas en manos de sociedades científicasy obtienen márgenes de beneficio que rondan el $40 \%$. Su modelo de negocio se basa en no remunerar el trabajo de las autoras y revisoras (investigadoras todas ellas) y en cobrar subscripciones, cada vez más caras, a las instituciones académicas en que trabajan. Esta lucrativa industria está incluso utilizando la actual demanda de Open Access para obtener aún más ingresos (Aibar, 2014).

\section{Cambios en la naturaleza del conocimiento científico}

La transformación neoliberal de la ciencia no sólo afecta a sus aspectos institucionales, sino que está teniendo un fuerte impacto en el mismo conocimiento científico: en su contenido, en sus métodos y en su orientación. Los procesos de mercantilización han tendido a favorecer los enfoques reduccionistas en biología, por encima de aquellos más holísticos. Con el fin de fomentar el control comercial sobre la innovación biológica y las herramientas de investigación, es necesario que la teoría identifique objetos discretos que puedan someterse a propiedad privada. Aquellas combinaciones de teoría y estudio empírico que, en cambio, enfatizan la complejidad y las interrelaciones entre fenómenos, resultan menos atrayentes bajo este prisma (Fochler, 2016; Mirowski, 2011).

El impacto es también notable en la selección de temas y objetos de investigación. Es conocido el caso de la investigación sobre nuevos medicamentos: se dedica cada vez más esfuerzo y financiación a encontrar curas y tratamientos para las enfermedades que afectan a los habitantes de países ricos y mucho menos a las enfermedades tropicales, que afectan a una población mucho mayor pero que habita países pobres - las denominadas enfermedades huérfanas. Otro fenómeno relacionado se observa en la presión que los lobbies farmacéuticos ejercen sobre los centros de investigación y los organismos regulativos, con objeto de reclasificar lo que hasta hace poco se consideraban simplemente estados o trastornos de la salud, como enfermedades e incluso epidemias que puedan ser, por lo tanto, medicalizadas más fácilmente y devenir objeto de tratamientos farmacológicos (Greenhalgh, 2016). 
Los estudios de diversos historiadores de la ciencia han puesto de manifiesto desde hace tiempo que el hecho de que Galileo trabajara sucesivamente para una universidad, a continuación, para la República de Venecia y, finalmente, en la corte del Gran Duque de la Toscana, tuvo una influencia directa en el tipo de conocimiento que produjo en cada etapa: en los objetivos y temas que persiguió y en los métodos y aproximaciones que utilizó (Biagoili, 1993; Pestre, 2005). La transformación neoliberal de la ciencia contemporánea es, análogamente, mucho más que un simple cambio en el modelo de financiación de la ciencia y está incidiendo de forma notoria sobre sus contenidos, sobre el propio conocimiento científico: desde sus métodos hasta sus resultados.

En el origen de las distintas fases históricas de la organización de la ciencia siempre ha habido un ámbito científico que ha actuado como punta de lanza o pionero del cambio. En la etapa de profesionalización de la ciencia, a finales del XIX, fueron la química y la ingeniería eléctrica; en la etapa de la guerra fría y el origen de la big science lo fue la física. El giro reciente hacia la privatización está, sin duda, liderado por el ámbito biomédico. La necesidad de reconfigurar la investigación con el fin de producir resultados en forma de elementos discretos comercializables está afectando, por encima de todo, al ámbito biomédico y es en este terreno donde se están realizando la mayoría de estudios y análisis para averiguar el alcance y trascendencia de estas transformaciones. En cambio, las ciencias sociales y las humanidades han recibido mucha menos atención, posiblemente porque tradicionalmente han tenido vínculos menos intensos con el ámbito productivo y la actividad económica. En este trabajo nos centraremos en el ámbito de las denominadas Humanidades Digitales.

Aunque, como veremos, se trata éste de un ámbito difícil de caracterizar, de aparición reciente - bajo esa denominación - y que incluye contribuciones muy diversas, es posible identificar algunos elementos y rasgos recurrentes que son característicos de la transformación neoliberal de la ciencia contemporánea en otras áreas. No se trata, evidentemente, de una tendencia que afecte a la totalidad de iniciativas y desarrollos de las Humanidades Digitales, pero sí que resulta suficientemente patente en muchos discursos programáticos, como para ser tenida en cuenta.

\section{5. ¿Humanidades digitales o estudios literarios digitales?}

Es difícil entender qué son las Humanidades Digitales. Algunos autores no dudan en considerar el término como una de esas expresiones de moda (buzzwords) que se repiten constantemente, connotando modernidad o innovación, pero que en realidad no tienen un contenido claro ni definen un área de investigación o trabajo académico específico (Svensson, 2010). Si se analizan los títulos de los trabajos que se presentan en congresos o publicaciones bajo el emblema de Humanidades Digitales, la tremenda dispersión temática es lo primero que salta 
a la vista. Más que un conjunto coherente de prácticas, uno tiene la impresión de observar una amalgama heterogénea de experiencias sin más coherencia que una cierta intersección entre temas o cuestiones vagamente humanísticas y cualquier desarrollo técnico digital. El significado del término parece poder incluir prácticamente cualquier cosa, "from media studies to electronic art, from data mining to edutech, from scholarly editing to anarchic blogging, while inviting code junkies, digital artists, standards wonks, transhumanists, game theorist $s$, free culture advocates, archivists, librarians, and edupunks under its capacious canvas" (Ramsay, 2013, 239).

Esta indefinición contrasta con la efervescencia de eventos (congresos, seminarios, cursos) e incluso desarrollos institucionales (departamentos, centros y grupos de investigación) que han utilizado ese término en los últimos años. A pesar de la sempiterna crisis de las humanidades, las Humanidades Digitales parecen un área floreciente que vive al margen de los recortes públicos y las restricciones presupuestarias de los últimos ańos y que, muy al contrario, parece haberse convertido en una verdadera industria de iniciativas y proyectos de todo tipo. Resulta sintomático, en ese sentido, que una de las pocas convocatorias abiertas para proyectos de investigación que subsisten en Cataluña, el programa Recercaixa, financiado enteramente por la entidad bancaria Caixabank y que sólo mantiene dos líneas temáticas en el terreno de las humanidades, destine una de ellas a las Humanidades Digitales — la otra es para la filosofía.

A pesar de la indefinición que se constata al examinar proyectos o textos que se autoadscriben al terreno de las Humanidades Digitales, existen muchos trabajos académicos en el área que comienzan ofreciendo alguna forma de definición o caracterización más o menos precisa. Una de las que aparece de manera más frecuente identifica dos elementos básicos. Por un lado, la aplicación de herramientas digitales a las humanidades y, por otro, la reflexión sobre la tecnología — sobre los cambios sociales y culturales que causa- desde las humanidades (Svensson, 2010; Rodríguez Yunta 2014, 453).

Después de haber examinado muchas comunicaciones presentadas en diversos congresos del ámbito, documentos programáticos de instituciones relevantes, artículos y proyectos destacados por los mismos promotores y representantes del campo, hay que señalar un par de cosas importantes de esta definición. A pesar de lo que muchos discursos programáticos sugieren, las Humanidades Digitales no incluyen a "todas" las humanidades, ni siquiera a una mayoría; es más, ámbitos humanísticos tan importantes como la filosofía no acostumbran a tener ningún tipo de presencia y si la tienen es residual. La gran mayoría de contribuciones provienen, de hecho, de un único ámbito, los estudios literarios - $\mathrm{y}$, por ello, los grandes profetas y apologetas, así como sus precursores iniciales, son académicos claramente adscritos a él一. Este desequilibrio, que varía internacionalmente, es particularmente evidente en Espańa (Fiormonte 2014, 12). La expresión Humanidades Digitales produce, por lo tanto, esta primera confusión: sería mucho más 
acertado hablar de 'estudios literarios digitales', con una presencia minoritaria de otras disciplinas. No es una confusión casual, como veremos, y muestra una tendencia a la hipérbole, endémica en este campo.

\section{Tecnocentrismo y determinismo tecnológico}

En segundo lugar, "la reflexión crítica sobre la tecnología y sus efectos sociales", desde las humanidades, resulta difícil de encontrar en el terreno de las Humanidades Digitales. A pesar de que el último congreso de Humanidades Digitales Hispánicas tiene como uno de sus objetivos explícitos promover "la reflexión crítica sobre la sociedad digital o sobre las tecnologías digitales desde las humanidades", examinado la totalidad de las ponencias presentadas en las sesiones plenarias no puede encontrarse ni una sola dedicada directamente a ese fin. De hecho, las Humanidades Digitales parecen vivir de espaldas a las grandes tradiciones de análisis y reflexión crítica y sistemática sobre la tecnología que se han producido, durante las últimas décadas, desde la filosofía, la historia, la antropología, la sociología o la economía.

Lejos de recoger las enseñanzas de esta vasta tradición humanística contemporánea o de promover nuevas líneas de análisis, muchos trabajos adoptan más bien la imagen convencional de la tecnología con dosis variables de determinismo tecnológico (Aibar, 2010), ideología californiana (Barbrook y Cameron, 1996) y solucionismo tecnológico (Morozov, 2015). En primer lugar, el uso de las TIC parece promoverse como fin en sí mismo. No tanto como instrumento para responder con mayor solidez las preguntas de investigación o para formular nuevas y mejores preguntas, sino porque su uso y, en general, la innovación tecnológica, se ven como factores intrínsecamente positivos. Las Humanidades Digitales se entienden como una suma de "tecnología-innovación-humanismo" (Rodriguez Yunta $2014,457)$ en la que el orden de los elementos no parece casual. La innovación tecnológica se considera políticamente neutra y una especie de magnitud escalar, sin dirección. Por ello no suelen hacerse distinciones significativas entre tipos de aplicaciones digitales o entre clases de plataformas. Todo desarrollo tecnológico en el ámbito digital parece igualmente encomiable, ya se trate de redes sociales propietarias como Facebook o Twitter, o de redes sociales académicas privadas como Research. Gate, Academia.edu o Mendeley (Serantes, 2016), que, en realidad y a pesar de su engañoso nombre, han sido creadas por empresas de capital riesgo con el objetivo de comerciar con los datos que los científicos introducen.

La tecnología es, sin duda, el motor primordial y la esencia explícita e implícita de las Humanidades Digitales. La tecnología protagoniza un cambio de paradigma (Lucia, 2012) que afecta, no sólo a las herramientas de investigación de las humanidades, sino a su misma esencia, a sus aspectos conceptuales. Se utilizan diferentes expresiones para enfatizar la naturaleza radical y revolucionaria de este cambio tecnológico: se habla de "revolución digital", de "transformación digital 
a una velocidad vertiginosa" (González-Blanco, 2016, 80), de nueva "revolución industrial" o de "revolución informática". Aunque se trata de denominaciones fuertemente cuestionadas por historiadoras y sociólogas de la tecnología, su uso tiene un claro objetivo estratégico: "the language here is the language of scholarship, but the spirit is the spirit of salesmanship - the very same kind of hyperbolic, hard-sell approach we are so accustomed to hearing about the Internet, or about Apple's latest utterly revolutionary product" (Kirsh, 2014).

En cualquier caso, el mensaje es que se trata de un cambio inexorable, inevitable e investido de una gran dosis de fatalismo, frente al que sólo queda sumarse sin discusión. Quien no lo haga o muestre una actitud crítica será acusado de obsoleto, conservador o anticuado (Grusin, 2014). Y, en este contexto, "parece mucho peor estar equivocado que resultar anticuado" (Luri, 2015, 7).

\section{De la torre de marfil al mercado}

Las Humanidades Digitales se autoproclaman la única salida plausible para las humanidades: o tomamos el camino que nos abren o estaremos condenados a la irrelevancia social y a la marginalidad académica. Se utiliza a menudo la imagen de la torre de marfil para evocar una situación de absoluta desconexión con el entorno social y cuestionar el elitismo del humanista tradicional (Aiden y Michel, 2014, 5). En contraste con esta situación, las Humanidades Digitales permiten construir una nueva (por casi inexistente hasta ahora) relación entre empresa y humanidades, entre el ámbito del mercado y la torre de marfil de los humanistas. Se trata, en fin, de hacer que las humanidades sean relevantes en el mercado (tanto por sus "productos", como por el tipo de graduados que formarán). Las Humanidades Digitales constituyen la salvación de las humanidades puesto que "posibilitan un nuevo acceso al mercado de todo un sector que parecía abocado a no encontrar su hueco en un mercado laboral no docente" (González-Blanco, 2016, 91). Para Aiden y Michel, de la misma forma que el telescopio de Galileo sirvió para descubrirnos nuevos e inimaginados mundos, la poderosas lente tecnológica del big data "is going to change the humanities, transform the social sciences and renegotiate the relationship between the world of commerce and the ivory tower" $(2014,5)$.

Dejando de lado el carácter hiperbólico de estas afirmaciones - muy habitual en el terreno de las Humaniades Digitales - es remarcable la similitud entre este discurso y dos de los rasgos básicos de la aproximación neoliberal al conocimiento científico. En primer lugar, es necesario acabar con la autoridad epistemológica de los expertos y del conocimiento experto académico (Hayek, 1949) - el rasgo político-epistémico clave de la era Trump y la "post-verdad". En segundo lugar, el conocimiento experto debe supeditarse al mercado que, como verdadero procesador de ideas, es el único medio genuino y "democrático" de obtener legitimidad (Mirowski, 2011, 324). El ataque populista al sabio ilustrado debe 
conducir a la adaptación sumisa del conocimiento a las leyes del mercado. De la torre de marfil al mercado: ese es el camino. "Relevancia social" se traduce por relevancia comercial y "transferencia a la sociedad" por colaboración con empresas.

Pero para salir al mercado con unas mínimas posibilidades de éxito es necesario realizar algunas transformaciones y movimientos previos que no resultan en absoluto triviales y que, efectivamente, pueden acabar afectando a la esencia del conocimiento humanístico: a sus formas y técnicas de investigación, a sus principios teóricos y, en general, a sus contenidos. Las Humanidades Digitales "han emergido gracias a la demanda de la sociedad de una puesta al día de todas las ciencias humanas o sociales y a la preocupación de sus profesionales de buscar salidas visuales a sus contenidos, tan denostados por el público en general. Se necesitaba urgentemente una renovación en la forma de vender nuestros productos" (López Cuadrado, 2015).

Esta cita pone en evidencia dos de las estrategias básicas de las Humanidades Digitales en su asalto al mercado. Por un lado, la necesaria conversión del conocimiento humanístico en unidades comercializables, es decir, "productos" - con la consiguiente colonización del ámbito por la terminología empresarial. Pero ¿cómo convertir el conocimiento humanístico, plagado de 'aproximaciones teóricas', 'críticas', 'marcos conceptuales', 'visiones del mundo', etc. en productos fácilmente mercantilizables? La estrategia que nos sugiere la cita es, en efecto, un rasgo habitual de las Humanidades Digitales: la preferencia por los productos "visuales". El énfasis se pone en producir visualizaciones atractivas, imágenes, vídeos, animaciones o representaciones que consigan atraer la atención del público. El formato tradicional del trabajo humanístico, el texto escrito, resulta anticuado e inapropiado para el nuevo objetivo comercial: "the 8-page essay and the 25page research paper will have to make room for the game design, the multi-player narrative, the video mash-up, the online exhibit and other new forms and formats" (Burdick et al., 2012, 24).

\section{8. "Hacer cosas" y soslayar la crítica}

Una de las expresiones que más llaman la atención en los algunos discursos programáticos de las Humanidades Digitales es la que enfatiza la voluntad de "hacer cosas" — make things (Chun et al., 2016; Grusin, 2014)—. La expresión se refiere a la vocación productiva y constructiva - en sentido literal- de las Humanidades Digitales, a su interés en obtener resultados tangibles (en forma de datos, visualizaciones o archivos digitales) y comercializables. Para entender completamente su significado, sin embargo, es necesario tener en cuenta a qué se opone. Y el punto de mira se sitúa, curiosamente, sobre las mismas humanidades, o mejor dicho sobre el ámbito originario de las humanidades digitales, los estudios literarios y sobre una forma particular de acometerlos. 
Es muy ilustrativo, en este sentido, observar el tipo de aproximación a los estudios literarios que imperaba en el departamento de Inglés de la Universidad de Virginia —uno de los enclaves originarios, según muchos autores, de las Humanidades Digitales-. Este departamento era conocido por su inclinación mayormente anti-interpretativa y fundamentalmente contraria a la denominada escuela francesa, dominante en muchos departamentos de inglés en los EE.UU. Esta escuela era denostada, por algunos miembros notorios del departamento virginiano, tanto por lo que se consideraba una tendencia excesiva a la abstracción, como por su inclinación hacia la crítica en clave política y social. Estas figuras defendían, en cambio, una tendencia más conservadora, pro-canon, anti-postmoderna y, en cualquier caso, contraria a la politización de los estudios literarios. "Hacer cosas" se convirtió en este contexto en un alegato contra los enfoques prioritariamente críticos y la interminable interpretación política a que conducían (Allington, Brouillette y Golumbia, 2016).

En buena parte las Humananidades Digitales han desplazado las visiones críticas y políticamente disidentes en favor de la construcción de herramientas y archivos digitales, en consonancia con una "creciente desvinculación de las actividades humanísticas respecto a un proyecto colectivo de emancipación, capaz de dar una respuesta suficiente al proyecto del capitalismo cognitivo" (Garcés, 2017, 71). En una suerte de giro postcrítico lo prioritario debe ser ahora la producción de datos, visualizaciones, materiales digitales o software; la interpretación es secundaria, inexistente o, simplemente, indeseable. Las Humanidades Digitales, por lo menos en este tipo de enfoque, acaban mostrando un tono sorprendentemente antihumanistico.

De hecho, un vistazo al programa académico de un título universitario de Experto profesional en Humanidades Digitales muestra todo un conjunto de competencias y contenidos tecnológicos diversos (edición digital de textos, lenguajes de programación, bases de datos, procesamiento de imágenes, etc.) y ni un solo curso con contenido mínimamente humanístico.

La centralidad que se otorga a la tecnología es tal que se considera prioritaria la "necesidad de tecnologizar los proyectos, las infraestructuras y, sobre todo, de formar a toda una comunidad científica de base humanística en unos conocimientos tecnológicos específicos adaptados a sus propias necesidades” González-Blanco $(2016,80)$. Lo que hasta ahora eran funciones de asistencia técnica para humanistas, pasan a convertirse en el núcleo de lo que debería ser su formación.

Además, como la tarea de formar a "toda la comunidad científica humanística" parece francamente poco factible, muchos proyectos en Humanidades Digitales acaban de hecho subcontratando las tareas técnicas a alguna de las muchas empresas privadas que han surgido en este ámbito (muchas de ellas lideradas 
por antiguos académicos). En un proceso similar al ocurrido en las ciencias biomédicas, se abre así la puerta a la privatización creciente de las herramientas de investigación.

\section{Datos e interpretación}

El énfasis en la producción de datos y el rechazo a los enfoques excesivamente interpretativos conduce a una relación particular entre datos e investigación. Si se examinan diferentes proyectos de investigación en Humanidades Digitales basta analizar aquellos que se destacan en algunos laboratorios, departamentos o sitios web del ámbito - sorprende la casi total ausencia de preguntas de investigación. Las pocas que aparecen resultan puramente descriptivas y suponen una versión banalizada o simplificada del tipo de problemas y preguntas que asociamos a las disciplinas humanísticas, incluso si nos restringimos al terreno de los estudios literarios.

En este sentido, es interesante comentar la propuesta metodológica de Franco Moretti, profesor de crítica literaria de tradición marxista en Stanford. Aunque Moretti proviene de la corriente metodológica interpretativa denominada close reading, en un momento dado se interesó por la posibilidad de utilizar métodos cuantitativos, normalmente asociados a las ciencias sociales, en el terreno literario. Aunque ya existía una cierta tradición de estudios cuantitativos aplicados al análisis de la literatura, el acierto de Moretti fue, por un lado, dar a conocer esas técnicas en revistas y publicaciones más próximas al ámbito humanístico y, por otro, conceptualizarlo con el término distant reading que sugería la posibilidad de analizar grandes cantidades de datos, mediante programas de ordenador y un cierto aparataje estadístico pero "sin necesidad de leer" las obras implicadas (Allington, Brouillette y Golumbia, 2016; Kirsch, 2014).

Moretti no se autoposicionó inicialmente como "humanista digital" pero sus contribuciones en torno al distant reading han sido recibidas con gran entusiasmo desde las Humanidades Digitales, muchos de cuyos representantes las consideran ejemplos paradigmáticos de la nueva corriente (González-Blanco, 2016, 80). Efectivamente, el denominado distant reading supone un cambio metodológico que va plenamente en la línea de lo que hemos señalado más arriba. En primer lugar, descansa en la primacía de los datos sobre la interpretación. Los datos no se utilizan para responder preguntas previas, sino que generan "ellos mismos", los patrones o regularidades que constituyen el análisis. En segundo lugar, la tecnología adquiere aún más protagonismo. No se trata únicamente de que los instrumentos informáticos nos ayuden a generar, agregar y ordenar grandes cantidades de datos, sino que son también los mismos programas informáticos los que nos proporcionan los análisis relevantes, sugiriendo recurrencias, pautas o modelos.

Este punto de vista sobre la relación entre datos y teoría descansa en una visión ciertamente naif y neopositivista de la naturaleza de los datos. Los datos 
se ven así como entidades "neutras", ateóricas, no construidas, aproblemáticas, capaces de "hablar por sí mismas" y de inducir, casi automáticamente, análisis teóricos así como las propias preguntas de investigación. Cualquier socióloga o filósofa de la ciencia sabe que eso no es así, en absoluto, ni siquiera en las ciencias naturales o "duras".

\section{Conclusiones}

La colonización de la investigación científica por parte de intereses privados está siendo promovida y potenciada por políticas científicas neoliberales que interpretan la relevancia social de la ciencia como simple transferencia al sector empresarial privado. Se trata de políticas científicas que incentivan el uso de patentes para favorecer la comercialización del conocimiento, que, simultáneamente, promocionan los sistemas de evaluación de la investigación puramente cuantitativos, promoviendo de forma explícita la competitividad por encima de la colaboración ("colaborar para competir mejor" nos dicen) y que, mediante las llamadas políticas de la excelencia, concentran cada vez más los recursos en unos pocos investigadores y centros de investigación, depauperando a pasos agigantados el resto del sistema universitario público (Mirowski, 2011; Quintanilla, 2012).

El giro neoliberal en la ciencia contemporánea está teniendo consecuencias muy importantes, no sólo en la organización y la gestión de la ciencia y las instituciones académicas, sino en las formas en que se producen los conocimientos científicos: en el tipo de resultados que se generan, en los métodos que se consideran más legítimos o en los enfoques teóricos más secundados. La mayoría de estudios que intentan analizar este tipo de cambios en el "contenido" de la ciencia, se han centrado prioritariamente en las ciencias naturales y "duras" y, en especial, en el ámbito biomédico - la punta de lanza en esta nueva fase histórica de la ciencia. Nuestro objetivo ha sido, en este sentido, mostrar cómo el terreno de las humanidades también está siendo afectado por fenómenos similares. Nos hemos ocupado de las denominadas Humanidades Digitales, un ámbito que, a pesar de su indefinición y vaguedad, representa un área ciertamente floreciente en cuanto a publicaciones, financiación e iniciativas institucionales.

Nuestro análisis no tiene voluntad exhaustiva ni pretendemos que las tendencias o principios programáticos que hemos identificado tengan validez para la amplia variedad de enfoques y desarrollos que suelen adscribirse a las $\mathrm{Hu}-$ manidades Digitales. Tampoco constituyen, sin embargo, aspectos residuales o minoritarios. Su presencia es lo suficientemente importante en algunos de los textos, autores e instituciones que se consideran emblemáticos en el área, como para merecer ser tenidos en cuenta.

El énfasis en la producción de resultados que puedan ser fácilmente reutilizados por la industria cultural y la insistencia en establecer vínculos con el mercado y favorecer la comercialización del conocimiento, constituyen rasgos nucleares 
del giro neoliberal en la ciencia actual, que condicionan el contenido mismo del conocimiento científico y los métodos utilizados para generarlo. La propensión a "tecnologizar" los proyectos de investigación y a otorgar a la tecnología —y a la formación técnica - un papel central en el nuevo panorama académico, resuenan con la tendencia tecnocrática neoliberal a convertir problemas pedagógicos y, en general, cuestiones socio-económicas o políticas, en problemas puramente tecnológicos que el mercado estará en condiciones de resolver.

No es de extrañar que los gestores universitarios (y algunas entidades financiadoras) estén más entusiasmados con las Humanidades Digitales, que los propios humanistas. El mantra de producir datos, visualizaciones, archivos, etc., encaja a la perfección con las evaluaciones cuantitativas de la productividad científica que la gestión y evaluación actuales de la investigación requieren. El éxito de las Humanidades Digitales tiene mucho que ver con su compatibilidad con el nuevo modelo de universidad neoliberal, donde la capacidad de establecer vínculos con el mercado y conseguir fondos privados/empresariales es clave.

\section{Referencias bibliográficas}

Aibar, E. (2010). A Critical Analysis of Information Society Conceptualizations from an STS Point of View. En Cognition, Communication, Co-operation, 8(2), 177-182.

Aibar, E. (2014). Ciència oberta, encerclament digital i producció col-laborativa. En T. Iribarren, O. Gassol y E. Aibar (Eds.), Cultura i tecnologia: els reptes de la producció cultural en l'era digital (pp. 99-120). Lleida: Punctum.

Aiden, E. y Michel, J. B. (2014). Uncharted: Big data as a lens on human culture. London: Penguin.

Allington, D., Brouillette, S. y Golumbia, D. (2016, 1 de mayo). Neoliberal Tools (and Archives): A Political History of Digital Humanities. Los Angeles Review of Books, obtenido de https://lareviewofbooks.org/article/neoliberal-tools-archives-political-history-digital-humanities/

Barbrook, R. y Cameron, A. (1996). The Californian ideology. Science as Culture, 6(1), 44-72.

Biagioli, Mario (1993). Galileo Courtier: The Practice of Science in the Culture of Absolutism. Chicago: University of Chicago Press.

Burdick, A., Drucker, J., Lunenfeld, P., Presner, T., y Schnapp, J. (2012). Digital_Humanities. Cambridge, MA: MIT Press.

Chun, W., Grusin, R., Jagoda, P., y Raley, R. (2016). The Dark Side of the Digital Humanities. En Gold M. \& Klein L. (eds.), Debates in the Digital Humanities (493-509). Minneapolis: University of Minnesota Press. 
Dardot, P. y Laval, C. (2014). The new way of the world: On neoliberal society. London: Verso Books.

David, P. A. (2004). Understanding the Emergence of 'Open Science' Institutions: Functionalist Economics in Historical Context. Industrial and Corporate Change, 13(4), 571-589.

Fiormonte, D. (2014). Digital Humanities from a Global Perspective. Laboratorio dell'ISPF, XI. DOI: 10.12862/ispf14L203.

Fochler, M. (2016). Variants of Epistemic Capitalism: Knowledge Production and the Accumulation of Worth in Commercial Biotechnology and the Academic Life Sciences. Science, Technology, \& Human Values, 41(5), 922948.

Foucault, Michel (2009). Nacimiento de la biopolítica. Madrid: Akal.

Garcés, M. (2017). Nueva ilustración radical. Barcelona: Anagrama.

González-Blanco, E. (2016). Un nuevo camino hacia las humanidades digitales: el laboratorio de innovación en humanidades digitales de la UNED. Revista Signa 25, 79-93.

Greenhalgh, S. (2016). Neoliberal science, Chinese style: Making and managing the 'obesity epidemic'. Social Studies of Science, 46(4), 485-510.

Grusin, R. (2014). The Dark Side of Digital Humanities: Dispatches from Two Recent MLA Conventions. Differences: A Journal of Feminist Cultural Studies, 25(1), 79-92.

Halffman, W. y Radder, H. (2015). The academic manifesto: From an occupied to a public university. Minerva, 53(2), 165-187.

Hayek, F. A. (1949). The intellectuals and socialism. The University of Chicago Law Review, 16(3), 417-433.

Kirsch, A. (2014, 2 de octubre). Technology Is Taking Over English Departments: The false promise of the digital humanities. New Republic, obtenido de https://newrepublic.com/article/117428/limits-digital-humanities-adam-kirsch

Lave, R., Mirowski, P. \& Randalls, S. (2010). Introduction: STS and neoliberal science. Social Studies of Science, 40(5), 659-675.

López Cuadrado, Ana (2015). El papel de los archivos en las Humanidades Digitales. Estado de la cuestión. Comunicación en el II Congreso de la Asociación de Humanidades Digitales Hispánicas: Innovación, globalización e impacto. Madrid.

Lucía Megías, J.M. (2012). Elogio del texto digital. Claves para interpretar el nuevo paradigm. Madrid: Fórcola. 
Luri, G. (2015). Pròleg. En Erasme de Rotterdam, Eduqueu els infants ben aviat en les lletres (pp. 7-30). Barcelona: Adesiara.

Mirowski, P. (2011). Science-Mart. Privatizing American Science. Harvard: Harvard University Press.

Morozov, E. (2015). La locura del solucionismo tecnológico. Madrid: Katz.

OECD (2015). OECD Science, Technology and Industry Scoreboard 2015: Innovation for growth and society. Paris: OECD Publishing.

Pellizzoni, L. \& Ylönen, M. (2012). Neoliberalism and technoscience: Critical assessments. Farnham: Ashgate Publishing.

Pestre, D. (2003). Science, argent et politique: un essai d'interprétation. París: Editions Quae.

Pestre, D. (2005). The Technosciences between Markets, Social Worries and the Political: How to Imagine a Better Future. En H. Nowotny, D. Pestre, E. Schmidt-Assmann, H. Schultze-Fielitz \& H. Trute (eds), The Public Nature of Science under Assault (pp. 29-52). Berlin: Springer.

Quintanilla, M. A. (2012). El pensamiento científico y la ideología de izquierdas. Pensamiento Crítico, artículo en línea. Consulta: 24-11-2017, http://www.pensamientocritico.org/migqui0312.htm.

Radder, H. (ed.) (2010). The Commodification of Academic Research. Pittsburgh: University of Pittsburgh Press.

Ramsay, S. (2013). Who's in and who's out. En E. Vanhoutte (ed.), Defining digital humanities: a reader (pp. 239-241). Surrey: Ashgate Publishing.

Rodríguez-Yunta, Luis (2014). Ciberinfraestructura para las humanidades digitales: una oportunidad de desarrollo tecnológico para la biblioteca académica. En El profesional de la información, 23(5), 453-462.

DOI: http://dx.doi.org/10.3145/epi.2014.sep.01

Serantes, M.A. (2016). Redes sociales académicas y humanidades digitales: un nuevo modelo interactivo en las relaciones humanas. Ponencia en el congreso Naturaleza humana 2.0, Universidad Pontificia de Comillas, Madrid.

Sismondo, S. (2009). Ghosts in the machine: publication planning in the medical sciences. En Social Studies of Science, 39(2), 171-198.

Svensson, P. (2010). The Landscape of Digital Humanities. En Digital Humanities Quarterly, 4 (1). 